\title{
Test of Tethered Deorbiting of Space Debris
}

\author{
Lorenzo Olivieri $^{1}$ (1) Andrea Valmorbida ${ }^{2} \cdot$ Giulia Sarego $^{1} \cdot$ Enrico Lungavia $^{3} \cdot$ Davide Vertuani $^{3}$. \\ Enrico C. Lorenzini ${ }^{1,2}$
}

Received: 23 August 2020 / Revised: 4 November 2020 / Accepted: 29 November 2020 / Published online: 14 December 2020 (c) The Author(s) 2020

\begin{abstract}
Current investigations on space tethers include their application to space debris deorbiting, specifically on the set of manoeuvres performed by a chaser tug to change the orbital parameters of a target body. Targets can be cooperative spacecraft at the end of their life or uncontrolled objects such as defunct satellites without clearly available capturing interfaces. In this latter case, a link joining tug and target may be misaligned with the target body inertia axes, influencing the attitude of both bodies; in case of rigid links, torques transmitted during tugging operations may overcome the tug attitude control system. This issue is clearly less significant in case of non-rigid connections, such as tethers; furthermore, with such connections the chaser can remain at a safe distance from the target during the whole deorbiting operation. On the other side, the initial phase of tethered space debris removal manoeuvres can be influenced by transient events, such as sudden tether tension spikes, that may cause longitudinal and lateral oscillations and, in case of resonance with the target attitude dynamics, could represent a serious issue for tug safety. In this paper it is proposed to provide the tug with a tether deployer mechanism capable to perform reel-in and reel-out, smoothing loads transmission to the target and damping oscillations. This concept is validated through a representative test campaign performed with the SPAcecRraft Testbed for Autonomous proximity operatioNs experimentS (SPARTANS) on a low friction table. A prototype of the deployer is manufactured and the deployment and rewind of a thin aluminium tape tether is proven. Test results include the verification of the tether visco-elastic characteristics with the direct measurement of spikes and oscillations and the estimation of the proposed system damping capabilities.
\end{abstract}

Keywords Space tethers $\cdot$ Deployment $\cdot$ Active debris removal $\cdot$ Low friction test




rendezvous and docking maneuvers [8], space tugging operations [9], and asteroids [10,11] and non-cooperative objects [12] capture.

To date, many investigations of space tethers are for applications in debris removal. The growing problem of space debris and their influence on the access to orbit became commonly recognized by the scientific community after the definition of the so-called "Kessler Syndrome" [13], introducing the risk of losing access to Earth orbit regions due to the constant growth of debris and the consequent catastrophic impacts cascade effect. Despite efforts to reduce new spacecraft influence on the debris environment (e.g., $[14,15])$, the recent plans for large constellations [16, 17] are constantly scrutinized and their short and long term influence on the space debris environment stability is under evaluation [18-20]. In this context the scientific community is evaluating further mitigation strategies, considering both the utilization of enhanced protections [21] and the implementation of post mission disposal (PMD) [22] and active debris removal (ADR) [23] operations. Among the different strategies for PMD and ADR [24], Electrodynamic Tethers (EDTs) have been investigated as a reliable and convenient solution in low-Earth orbit (LEO) [25, 26]. For further information on EDTs and the most recent evolution, the lowworking-function tethers (LWTs), see [27-30].

The advantages of tether systems are not limited to the disposal manoeuvre, as flexible connections between two modules reduce the loads transmitted between them with respect to solid joints. Furthermore, with such connections the vehicles involved can remain at a safe distance while maintaining a physical connection. For this reason, tethers have been also proposed for space tug configurations and both experimental investigation [31,32] and simulation activities [9, 33, 34] have been carried out.

In this context, the H2020 Future Emerging Technologies FET OPEN Project E.T.PACK-Electrodynamic Tether Technology for Passive Consumable-less Deorbit Kit [35] is currently investigating a number of technologies for PMD with EDTs [36, 37], including safe tether deployment [38]. In this paper, some preliminary tests on tether technologies for deorbiting are presented. In particular, a tether deployment and retrieval mechanism developed in the framework of ETPACK project is introduced. A breadboard prototype of the deployer was manufactured and the deployment and rewind of a thin aluminium tape tether were tested. Test results include the determination of the tether visco-elastic characteristics, the direct measurement of spikes and oscillations and the estimation of the proposed system damping capabilities. The prototype, while designed principally for the ETPACK kit tether deployment, can be employed also for tethered systems formation flight and space tugging, thanks to the capability to control both the tether deployment and retrieval.

\subsection{Tether Deployment Background}

One of the most challenging operations with tethers is the deployment phase, as several issues have to be taken into consideration, such as the libration stability during the process [39-41]. The most investigated approach, employed also on the TSS-1 demonstration [42], starts with releasing a tethered tip mass from the host spacecraft (i.e. the ETPACK deployer kit from the main spacecraft, or the tagging unit from a tugged satellite). Due to the negligible authority of gravity gradient forces acting on the system after separation, the tip mass is provided with an initial momentum to reel the tether out; once few hundreds of meters are deployed, the gravity gradient can become the leading driver in the deployment process. A continuous control of the tip mass is requested during the deployment, as uncontrolled oscillations can lead to system instability (see [43] for a more detailed description of stability conditions and range). To provide the requested stability, reference deployment trajectories can be defined and optimal values for the tip mass initial attitude and velocity can be computed; the values adopted for ETPACK are reported in [38].

The deployer shall therefore be able to follow the predefined deployment profiles by providing the requested initial momentum and by controlling the tape reel-out by passive or active control systems. Mechanical (springs [8, $44,45]$ ), electro-mechanical (deployed masts [46]) or propulsive (cold gas actuators $[38,47]$ ) systems have been proposed to provide the initial momentum, with the latter one selected for ETPACK. With regards to deployment mechanisms, they can be classified in three main categories: stationary spools, rotating reels, and folded "origami", depending on the tether stowing strategy; again, for this work a rotating reel configuration was selected.

Among the several in-space (e.g.: TSS-1 [42, 48], ProSEDS [49] - cancelled after Shuttle Columbia accident, YES II [50, 51], SEDS [52], SEDS II [53]) and ground verifications on deployment mechanisms ([44, $45,54]$ ), only the TSS-1 [48] and the STAR prototype [45] demonstrated the ability to reel in. More recently, the TEPCE CubeSat technology demonstrator was conceived and flown to deploy a 1-km-long tether; to date, confirmation of 500-m deployment is available [55]. The low success rate of tethered systems can be related to the complexity of deployment operations, in which minimal disturbances such as internal components friction can greatly influence the reel-out process up to stop completely the tether deployment. For this reason, the proposed deployer employs a system of motorized pulleys to control the tether motion and to decouple the internal mechanism dynamics from the external motion subjected to orbital dynamics. 
An important constraint for E.T.PACK deployer mechanism is the utilization of a tape tether instead of a round wire. This was first introduced by BETs project [28] due to tapes higher survivability to space debris impacts [56], their increased performance in collecting electrons and the faster deorbiting they provide. The utilization of a tape greatly affects the design of the deployer mechanism, as the bulk and shape of a tape coil is different from a wire one, as well as the extraction technology to deploy it.

\subsection{Paper Contents}

The remainder of this paper is organized as follows. Section 2 presents the deployer concept, while Sect. 3 introduces the experimental setup employed for tests reported and discussed in Sect. 4.

\section{Tether deployment module}

The deployer mechanism described in this work in the framework of the E.T.PACK project is sketched in Fig. 1. It consists of a Cold Gas Actuator (CGA) propulsive unit, employing two nozzles, a tank, and the relative fluidic system, and a deployment/reel-in subsystem, composed by a rotating reel, a system of pulleys to control the tape deployment, and a motor coupled to the reel to brake it during deployment and actuate it during reel-in operations. Thanks to the pulleys system, the proposed layout is able to decouple the tether dynamics outside of the deployment module from one of the internal mechanisms, leading to a safer deployment. With this configuration, the whole module can act as

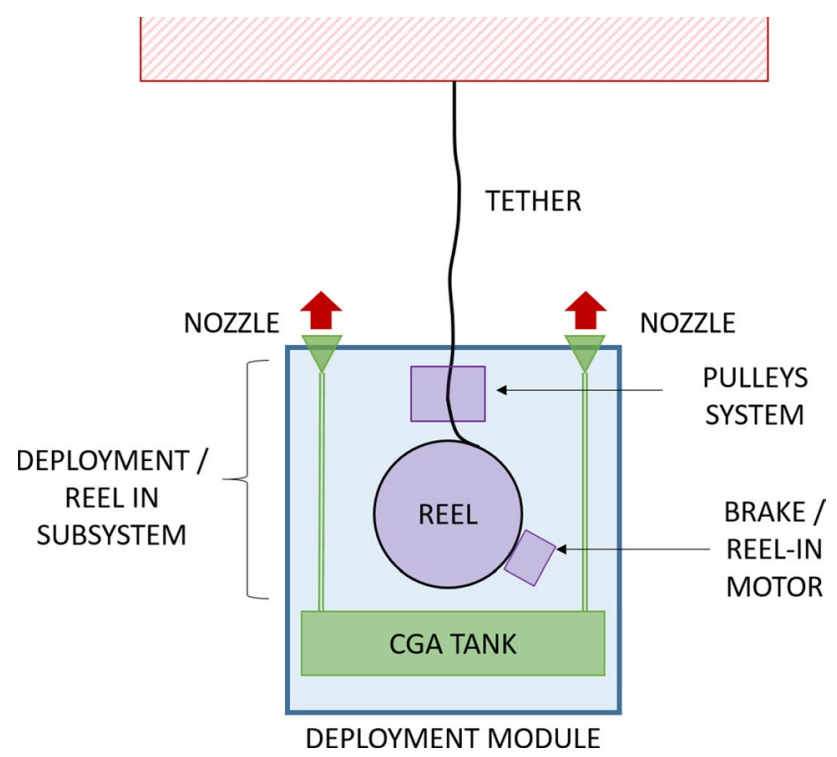

Fig. 1 Sketch of the proposed deployment module tip mass during the deployment, as well as perform the reelin operations by switching the motor function.

In more details, a tether deployment procedure (see [38] for E.T.PACK reference profiles) starts with the release of the module. In a first acceleration phase, the CGA provides enough momentum to the tip mass and keeps the tape in tension, while the pulleys system controls the tether deployment. In this phase, the brake is not active (i.e., the brake torque is negligible) letting the module to accelerate. After this stage the deployment motion is controlled only by the pulleys, the tip mass momentum, and, after a sufficient length of tape is deployed, by the gravity gradient. In this second phase, the tape velocity is not constant and while the pulleys can control the deployment profile, the reel rotation is controlled by the brake to avoid tape unwinding inside the module. On the contrary, rewind procedure requires the actuation of the reel; in this case the brake is switched to the motor and controls the reel-in procedure, while the pulleys system is not actuated.

The capability of the proposed module to deploy and retrieve a tether was assessed with a campaign of tests on a low-friction table, as described in the following sections. It is important to assess the early separation phase of deployment because the thrust direction depends on the attitude of the deployer module and this coupling effect must be analysed. Similarly, the tether retrieval requires further investigation because, due to the conservation of angular momentum, any attitude motion of the tip mass is amplified by the rewind process.

\section{Experimental Setup}

The experimental setup consists of a scaled deployment module (S-DM) mounted on board an air carriage system including three air bearings on its bottom part for a lowfriction motion on a low-friction test table.

The S-DM is shown in Fig. 2, with its main subsystems highlighted: the CGA, the deploy and reel-in subsystem, and the control and communication electronics. The whole module is painted in black to reduce the interference by multiple reflexions with the laboratory motion capture system.

The air carriage system and the test table are part of the SPARTANS facility [57-60], that can be seen in Fig. 3. The facility consists in a $3 \times 2 \mathrm{~m}$ test table and a spacecraft test mock-up (composed by a translational module and an attitude module); a Motion Capture (MC) system with 6 infrared cameras tracks the motion of the mock-up.

In the investigated configuration the S-DM is directly mounted on SPARTANS mock-up translational module replacing the original attitude module; the total mass of the free-floating system is about $25 \mathrm{~kg}$, comparable to the E.T.PACK mass. With this configuration, the S-DM acquires 




Fig. 2 Scaled deployment module with CGA (A-fluidic system, B-tank, C-nozzles), deployment and reel-in subsystem (D-rotating reel, E-brake/reel-in motor, F-pulleys system), and G-electronics

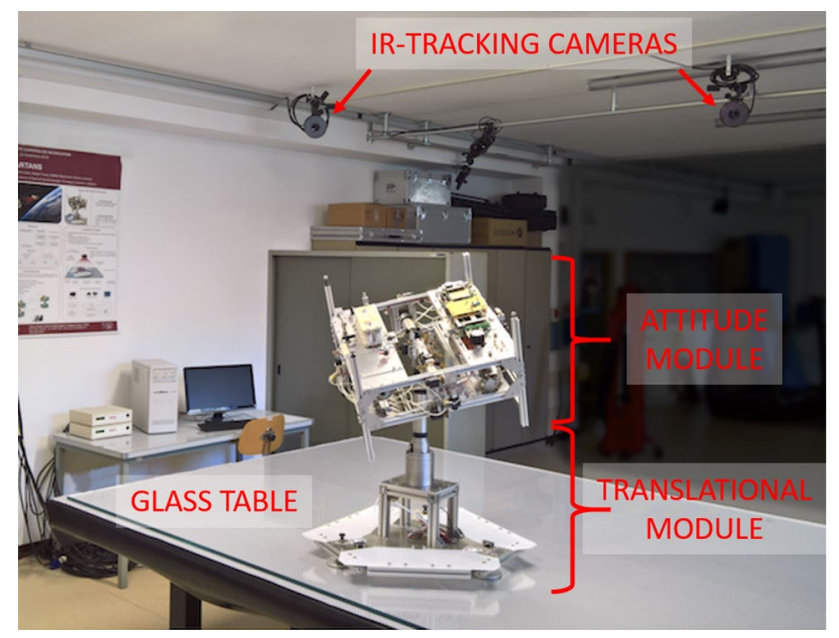

Fig. 3 SPARTANS test table and free-floating module

three degrees of freedom (DoF), the two translations on the test table and the rotation around the local vertical axis. The free end of the tether is constrained to a dedicated structure on one edge of the test table. The constraint on the tether end is an adequate approximation of a larger mother-satellite (i.e., with a mass ratio $>10$ ) that is unaffected by the transient loads transmitted by the tether during the operations simulated in the laboratory experiments. A load cell is placed on the interface between the tether and the fixed structure to assess the magnitude of the transmitted loads.

The S-DM is equipped with a Wi-Fi communication link; an external control station is employed to start experiments, log data and monitor the whole system.

This experimental setup allows the investigation of tether proximity operations (i.e., the early phase of deployment and the final stage of reel-in). Specifically, the coupling between translation and attitude dynamics was evaluated, as well as the effect of the CGA thrust on the deployment profile.

\section{Experimental Campaign}

The experimental campaign reported in this section consisted in four different activities: the verification of (1) the CGA thrusters force authority and (2) the tape mechanical characteristics and the tests of (3) deployment and (4) retrieval manoeuvers.

\subsection{Thrusters Authority}

The CGA thrusters are designed as simple convergent nozzles and a linear relation between inlet pressure and thrust force is expected. The inlet pressure is directly measured with an on-board sensor placed in the low-pressure section of the fluidic subsystem; the data live stream is transmitted through the Wi-Fi link to the control station. To evaluate the thrust, the S-DM is connected with a thin line of about $1.5 \mathrm{~m}$ to a load cell constrained to one vertex of the test table; independently from the mechanical characteristics of the line, once the system is in steady-state configuration (i.e. the S-DM does not present rotational and translational motions) the load cell measures the force actuated by the two thrusters on board the S-DM. Experimental results (red dots) are reported in Fig. 4 with a first-order fit (blue dashed line), indicating a linear relation between the inlet static pressure and trust authority, with a coefficient of determination $R^{2}=0.9997$.

The thrust levels are comparable to those required by the E.T.PACK reference deployment profile [38].

\subsection{Tape Mechanical Characteristics Verification}

The stiffness and damping of a flexible line can be verified by applying a constant tensile load in slack conditions and then measuring the line dynamic response in terms of tension spikes, oscillations, and damping. With the proposed experimental setup, the verification can be performed by constraining a section of the line with known length to a 


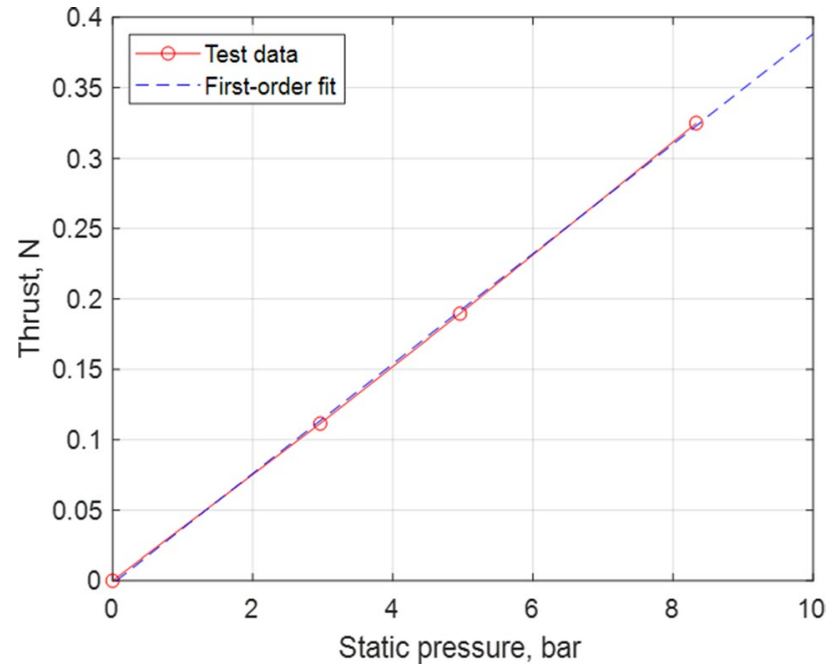

Fig. 4 Measured authority of one thruster (red) and linear fit (blue dashed lines)
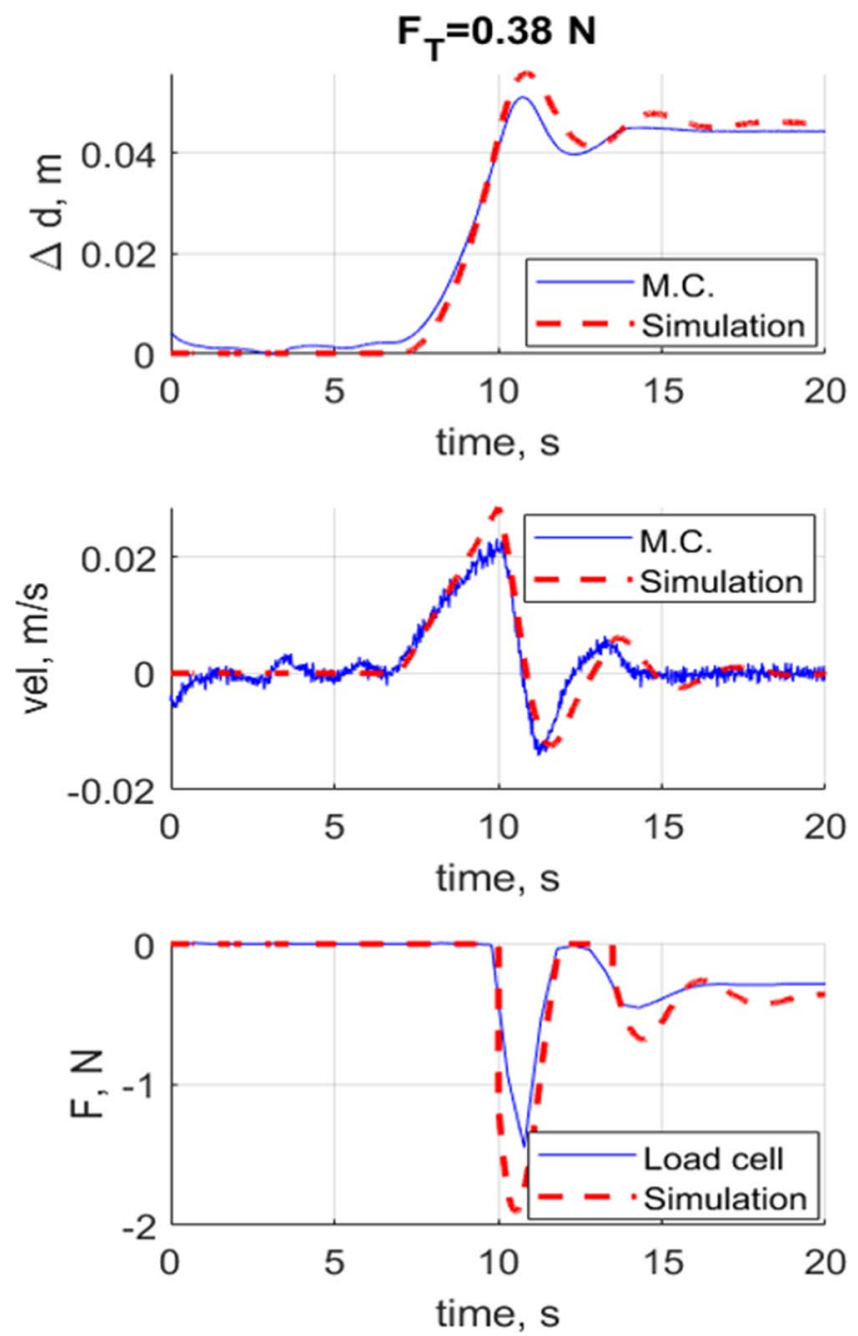

fixed structure and to the S-DM, and then actuating the S-DM thrusters with the line in the initial slack condition.

Due to the high stiffness of the employed aluminium tape (about $30 \mathrm{kN} / \mathrm{m}$ for a sample $1.5 \mathrm{~m}$ long), the verification is performed in two steps. First, the setup employed for thrusters authority verification is used to test the proposed method with a $1.5 \mathrm{~m}$ long polyamide line with a diameter of $0.3 \mathrm{~mm}$ (theoretical stiffness of $129 \mathrm{~N} / \mathrm{m}$ ); the S-DM motion detected with the MC system is reconstructed and compared to simulations, as well as loads measured with the load cell mounted on the constrained end. In a second phase, the line is substituted with the aluminium tape and the test is repeated with the same parameters but without the load cell, whose stiffness is comparable to that of the tape and might therefore invalidate the experiment. This approach allows a first validation of the proposed method with the elastic polyamide line and then the verification of the tape characteristics.
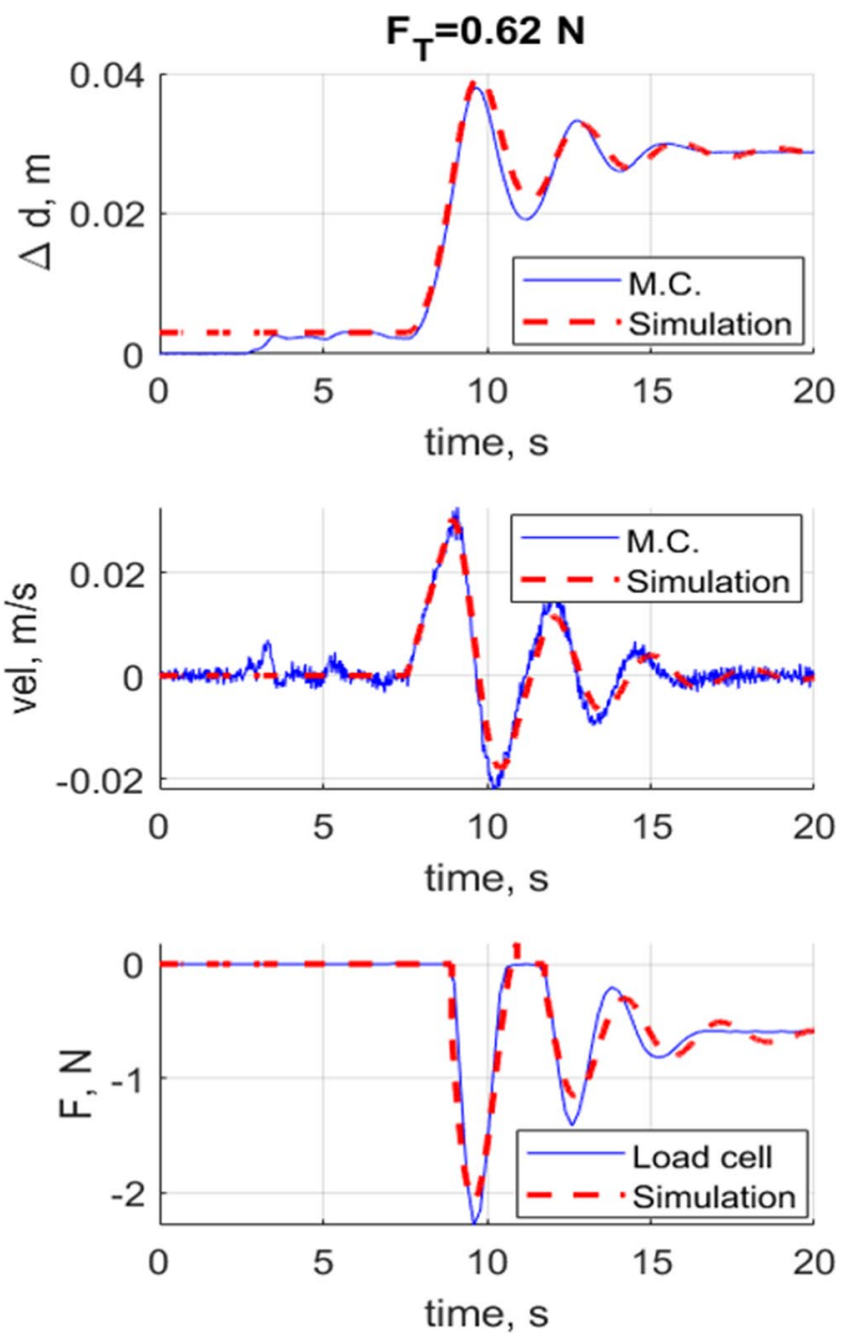

Fig. 5 Polyamide line mechanical characteristics verification with low (left) and high (right) thrust level: comparison of experimental data (solid blue lines) with simulations (dashed red lines). From top to bottom: S-DM translation, S-DM velocity, and force on the constraint 
Results for the polyamide line are reported in Fig. 5, for two different thrust conditions $(0.38$ and $0.62 \mathrm{~N})$. It can be seen that experimental data match simulations results; small discrepancies can be related to residual friction effects between the module and the test table.

Aluminium tape verification results can be seen in Fig. 6 . As previously mentioned, due to the higher stiffness of the tape with respect to the polyamide line the load cell is removed from the setup to avoid undesired effects. To match simulations with experimental data a high damping coefficient $(480 \mathrm{~N} \mathrm{~s} / \mathrm{m})$ is employed; the consequent damping ratio of 0.22 is higher than expected for aluminium alloys and can be related to memory effects of the tape sample undergoing testing.

\subsection{Deployment Manoeuvre}

The objective of this test is the verification of the deployer capability to follow a predefined reference deployment trajectory, in terms of tether length and length rate. While in operative configurations the deployment would last tens of minutes (up to $1 \mathrm{~h}$ in [38]), the test table available area limits it to less than $1 \mathrm{~min}$. Despite such constraint, the test allows to determine the behaviour of the deployment system and to verify the capability to follow a reference reel-out profile. Furthermore, the test aims to verify the capability of the deployment motor to follow a profile with a high initial acceleration as well as to monitor the effect of such acceleration on the S-DM dynamics. The reference profile, therefore, presents an initial acceleration of $7 \mathrm{~mm} / \mathrm{s}^{2}$ (about one-third of the in-orbit case value, as reported in [38]), and then a constant-velocity phase at $0.1 \mathrm{~m} / \mathrm{s}$. The other parameters (CGA thrust, S-DM mass) are comparable to the E.T.PACK reference ones [38].

Figure 7 reports the results of a deployment test, with the reconstructed trajectory from the MC system, the comparison of the deployment profiles with the reference ones, and some frames from the deployment video. It can be noted that the S-DM is capable to follow the pre-imposed deployment profile, with minimal discrepancies related to the dynamical response of the tape (small oscillations and spikes).

It can be noted that the S-DM is subjected to a small rotation on its centre of mass around the vertical axis, which is aligned to the tape coil axis: due to angular momentum conservation, the initial coil angular acceleration causes a small counter-rotation on the module. This issue will be addressed in future upgrades of the deployment hardware with a dedicated attitude control strategy modulating the module thrusters firing. It shall be underlined that the tape tension creates a restoring torque on the S-DM opposing the coil-induced torque, limiting the rotation to less than $45 \mathrm{deg}$.

\subsection{Reel-In Operations Example}

The capability to reel-in the tape is an important feature of the proposed system. Figure 8 reports an example of a reelin manoeuvre at a constant retrieval velocity of $0.02 \mathrm{~m} / \mathrm{s}$, with the reconstructed trajectory from the MC system, the reel-in and reel-in rate profiles, and some frames from the corresponding video. In this test after the initial acceleration, the coil rotation is constant; the thrusters are firing during the whole manoeuvre to maintain the tape in tension. Again,
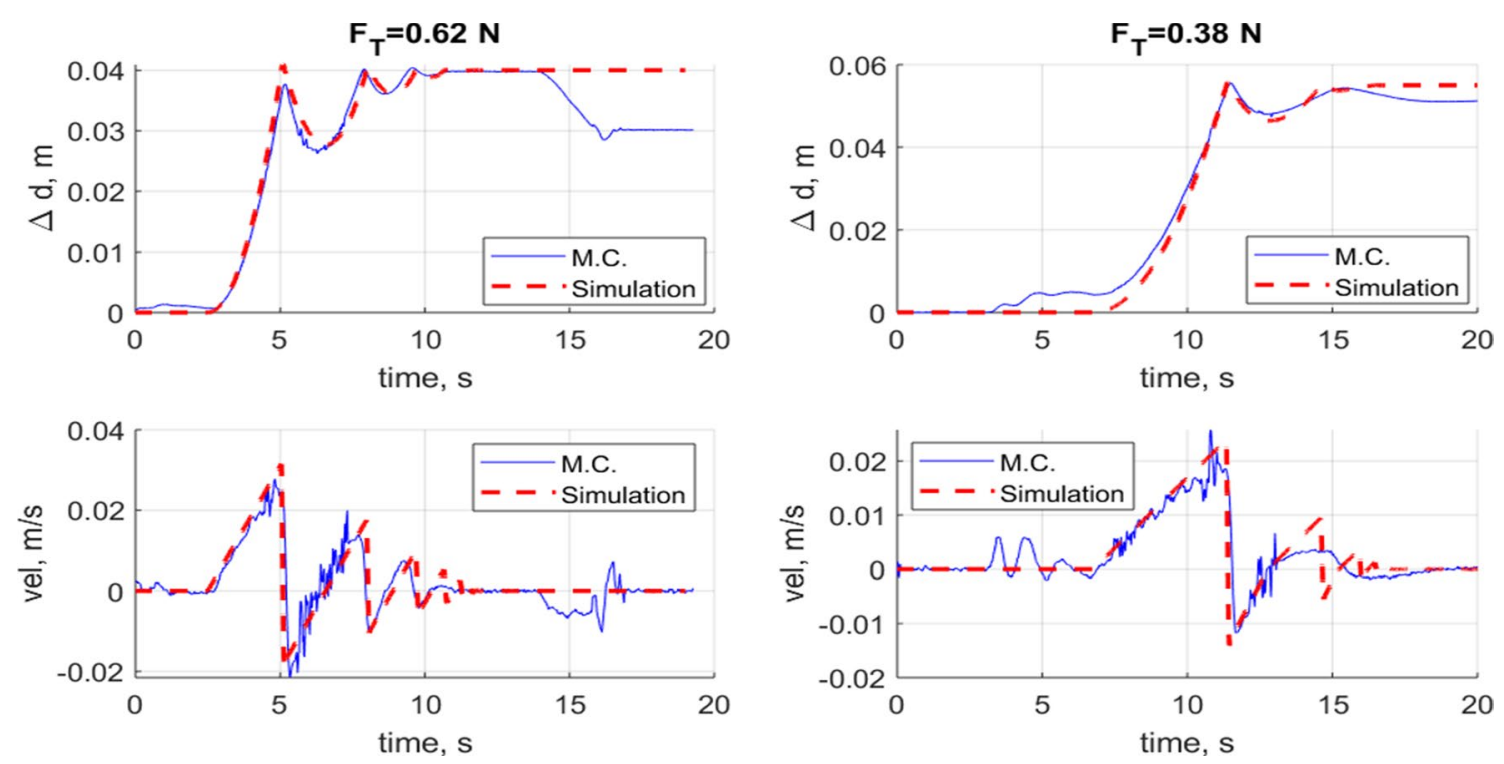

Fig. 6 Aluminium tape mechanical characteristics verification with low (right) and high (left) thrust level: comparison of experimental data (solid blue lines) with simulations (dashed red lines). From top to bottom: S-DM translation and S-DM velocity 



Fig. 7 Deployment test, with reconstructed module position from Motion Capture (top left), tether deployed length and velocity compared with reference profile (bottom left), and captured video frames (right)
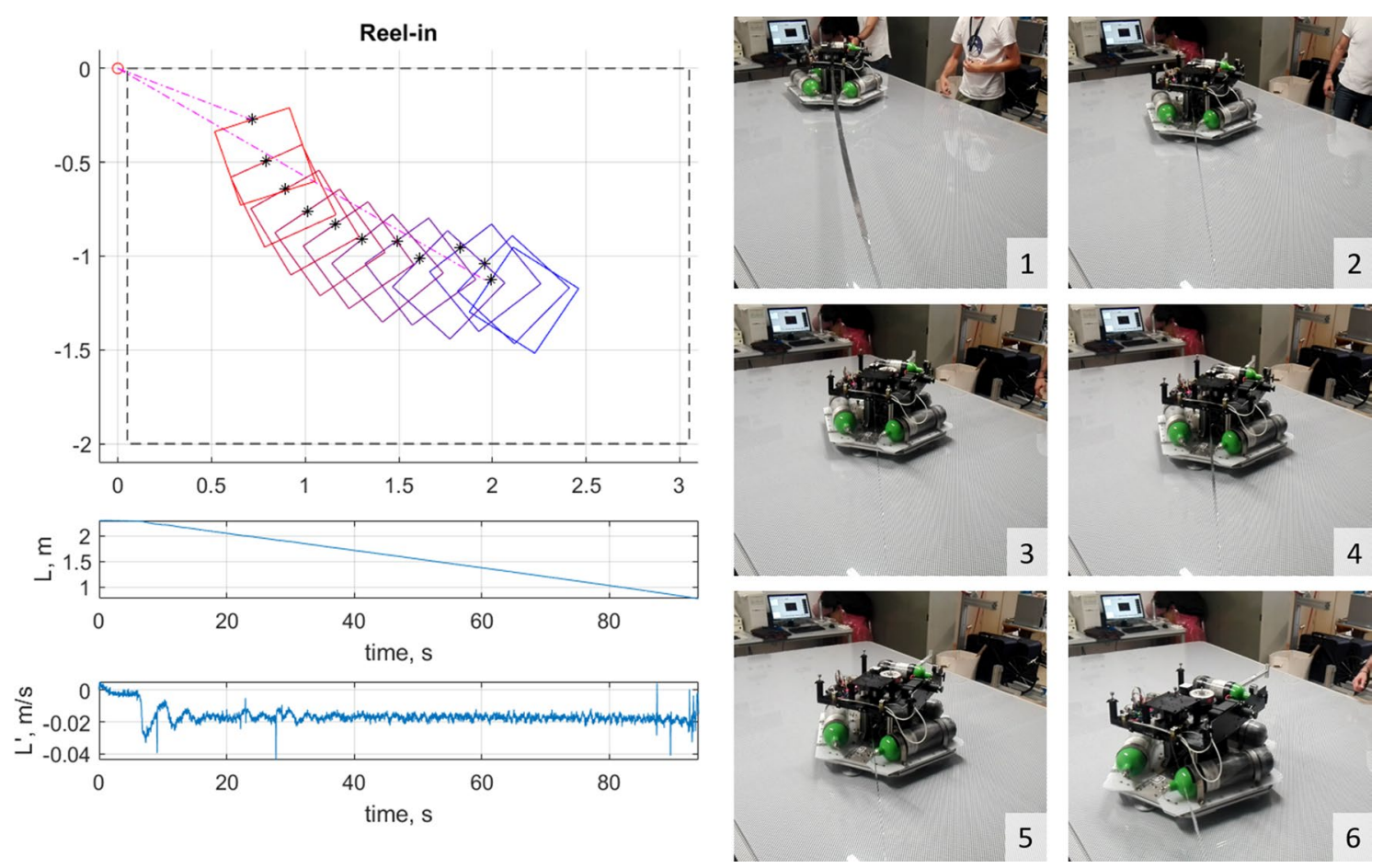

Fig. 8 Reel-in test, with reconstructed module position from Motion Capture (top left), tether deployed length and velocity (bottom left), and captured video frames (right) 
it can be noted an initial rotation of the S-DM due to the initial angular acceleration of the tape coil; as expected, the tape creates a small restoring torque opposing the module rotation.

This test demonstrates the proposed system general capability to reel-in at a constant velocity; further investigation will be performed to verify the capability to follow a reference retrieval profile and the possibility to perform it without the assistance of the propulsion system.

\section{Conclusions}

This paper presented the low-friction tests of a tether deployer and reel-in system developed in the framework of the H2020 ETPACK project. The system is based on a rotating coil concept, with a system of pulleys to control the deployment and a motor coupled to the coil to brake it during the reel-out and actuate it for the reel-in. The proposed system is integrated on a small module equipped with a cold gas system for propulsion purposes, and it is tested on the low-friction test table of the SPARTANS facility.

The first tests verified the cold gas system trust authority and the employed aluminium tape mechanical characteristics; it was found that a linear relation exists between the inlet static pressure and the thrust and that the aluminium tape presents a larger damping ratio than expected, due most likely to memory effects present in the tested tape sample.

A controlled deployment test demonstrated the system capability to reel-out the tape following a reference trajectory; it has been found that the angular acceleration of the tape coil affects the attitude of the deployment module but does not influence the deployment profile. Similarly, a reelin test demonstrated the capability of the proposed system to retrieve the tape.

It can be concluded that the proposed system demonstrated its desired features. Future works will focus on two main topics: the attitude control of the module during the manoeuvres will be implemented by modulating the thrusters firing and more complex deployment and retrieval operations will be tested.

Acknowledgements The authors wish to thank Mr Francesco Babolin, Mr. Mirco Bartolomei, and Mr Alex Caon for the support in the development of the experimental setup. This work was supported by the European Union's H2020 Research and Innovation Programme under Grant Agreement no. 828902 (E.T.PACK Project).

Funding Open access funding provided by Università degli Studi di Padova within the CRUI-CARE Agreement.

Open Access This article is licensed under a Creative Commons Attribution 4.0 International License, which permits use, sharing, adaptation, distribution and reproduction in any medium or format, as long as you give appropriate credit to the original author(s) and the source, provide a link to the Creative Commons licence, and indicate if changes were made. The images or other third party material in this article are included in the article's Creative Commons licence, unless indicated otherwise in a credit line to the material. If material is not included in the article's Creative Commons licence and your intended use is not permitted by statutory regulation or exceeds the permitted use, you will need to obtain permission directly from the copyright holder. To view a copy of this licence, visit http://creativecommons.org/licenses/by/4.0/.

\section{References}

1. Grossi MD, Colombo G (1978) Interactions of a tethered satellite system with the ionosphere. In: Wu ST (ed) Proceedings of the University of Alabama/NASA workshop on the uses of a tethered satellite system, pp 176-181

2. Cosmo ML, Lorenzini EC (1997) Tethers in space handbook. Resource online https://www.nasa.gov/centers/marshall/ pdf/337451main_Tethers_In_Space_Handbook_Section_1_2.pdf

3. Cartmell MP, McKenzie DJ (2008) A review of space tether research. Prog Aerosp Sci 44(1):1-21

4. Chen Y, Huang R, Ren X, He L, He Y (2013) History of the tether concept and tether missions: a review. ISRN Astron Astrophys. https://doi.org/10.1155/2013/502973

5. Chen Y, Huang R, He L, Ren X, Zheng B (2014) Dynamical modelling and control of space tethers: a review of space tether research. Nonlinear Dyn 77(4):1077-1099

6. Chung SJ, Adams D, Saenz-Otero A, Kong EM, Miller DW, Leisawitz D, Lorenzini EC, Sell S (2006) SPHERES tethered formation flight testbed: advancements in enabling NASA's SPECS Mission. Society of Photo-Optical Instrumentation Engineers (SPIE), Bellingham, $\mathrm{p}$ 62680B

7. Huang P, Zhang F (2020) Theory and applications of multi-tethers in space. Springer, Berlin

8. Olivieri L, Sansone F, Duzzi M, Francesconi A (2019) Ted project: conjugating technology development and educational activities. Aerospace 6(6):73

9. Mantellato R, Olivieri L, Lorenzini EC (2017) Study of dynamical stability of tethered systems during space tug maneuvers. Acta Astronaut 138:559-569

10. Mashayekhi MJ, Misra AK (2014) Optimization of tether-assisted asteroid deflection. J Guid Control Dyn 37(3):898-906

11. Feltrin F, Bettiol L, Olivieri L, Francesconi A (2017) Multibody tether concept for asteroids capture. In: 68th IAC, Adelaide, Australia, 25-29 September 2017

12. Olivieri L, Feltrin F, Valmorbida A, Mantellato R, Lorenzini EC, Francesconi A (2018) Enabling ENVISAT deorbiting: a multispacecraft mission for inspection, capture, and detumbling. In: 4S symposium, Sorrento, Italy, May 2018

13. Kessler DJ, Cour-Palais BG (1978) Collision frequency of artificial satellites: the creation of a debris belt. J Geophys Res Space Phys 83(A6):2637-2646

14. Inter-Agency Space Debris Coordination Committee (2007) IADC space debris mitigation guidelines

15. Stokes H, Akahoshi Y, Bonnal C, Destefanis R, Gu Y, Kato A, Kutomanov A, LaCroix A, Lemmens S, Lohvynenko A, Oltrogge D, Omaly P, Opiela J, Quan H, Sato K, Sorge M, Tang M (2019) Evolution of ISO's space debris mitigation standards. In: 1st International orbital debris conference (IOC), Sugar Land, TX, USA

16. Alvarez J, Walls B (2016) Constellations, clusters, and communication technology: expanding small satellite access to space. In: Aerospace conference, 2016 IEEE. IEEE, Big Sky, MT, USA, pp $1-11$ 
17. https://spaceflightnow.com/2020/04/21/spacex-modifies-starl ink-network-design-as-another-60-satellites-gear-up-for-launch/. Accessed July 2020

18. Karacalioglu AG, Stupl J (2016) The impact of new trends in satellite launches on the orbital debris environment. Online report. https://ntrs.nasa.gov/search.jsp?R=20160011184

19. Foreman VL, Siddiqi A, De Weck O (2017) Large satellite constellation orbital debris impacts: case studies of Oneweb and SpaceX proposals. In: AIAA SPACE and astronautics forum and exposition, Orlando, FL, USA

20. Olivieri L, Francesconi A (2020) Large constellations assessment and optimization in LEO space debris environment. Adv Space Res 65(1):351-363

21. Rossi A, Alessi EM, Schettino G, Beck J, Holbrough I, Schleutker T, Letterio F, Vicario de Miguel G, Becedas Rodríguez J, Dalla Vedova F, Stokes H, Colombo C, Gkolias I, Bernelli Zazzera F, Miguel Banos N, Walker S, Romei F, Tsiganis K, Skoulidou D, Stoll E, Schaus V, Popova R, Kim Y, Francesconi A, Olivieri L, Gerardin S (2019) The H2020 ReDSHIFT project: a successful European effort towards space debris mitigation. In: 70th International astronautical congress (IAC 2019), Washington, DC, USA

22. Henning GA, Sorge ME, Peterson GE, Jenkin AB, Mains D, McVey JP (2019) Parameterizing large constellation post-mission disposal success to predict the impact to future space environment. LPICo 2109:6037

23. Liou JC (2011) An active debris removal parametric study for LEO environment remediation. Adv Space Res 47(11):1865-1876

24. Shan M, Guo J, Gill E (2016) Review and comparison of active space debris capturing and removal methods. Prog Aerosp Sci 80:18-32

25. Sanchez-Arriaga J, Sanmartin JR, Lorenzini EC (2017) Comparison of technologies for deorbiting spacecraft from low-earth-orbit at end of mission. Acta Astronaut 138:536-542

26. Sato T, Kawamoto S, Ohkawa Y, Watanabe T, Kamachi K, Okubo $\mathrm{H}$ (2020) Performance of EDT system for deorbit devices using new materials. Acta Astronaut

27. Sanmartin JR, Martinez-Sanchez M, Ahedo E (1993) Bare wire anodes for electrodynamic tethers. J Propuls Power 9(3):353-360

28. Sanmartín Losada JR, Charro M, Lorenzini EC, Colombatti G, Roussel JF, Sarrailh P, Williams JD, Xie K, García de Quirós F, Carrasco JA, Rosta R, Zoest T, Lasa J, Marcos J (2012) BETs: propellant less deorbiting of space debris by bare electrodynamic tethers, let's embrace space, vol II. European Commission. ISBN:978-92-79-22207-8

29. Williams JD, Sanmartín JR, Rand LP (2012) Low-work-function coating for an entirely propellantless bare electrodynamic tether. IEEE Trans Plasma Sci 40(5):1441-1445

30. Sánchez-Arriaga G, Chen X (2018) Modeling and performance of electrodynamic low-work-function tethers with photoemission effects. J Propul Power 34(1):213-220

31. Mantellato R, Lorenzini EC, Sternberg D, Roascio D, SaenzOtero A, Becker M (2017) Simulation of a tethered microgravity robot pair and validation on a planar air bearing. Acta Astronaut 138:579-589

32. Benvenuto R, Lavagna M, Lunghi P, Pesce V, Bellanca A, Rafano S, Delahaye A, Pfeiffer L (2017) Tethered-tugs for active debris removal: microgravity experimental validation of dynamics and control. In: 7th European conference on space debris, ESA/ESOC, Darmstadt, Germany, 18-21 April 2017, pp 1-11

33. Aslanov V, Yudintsev V (2013) Dynamics of large space debris removal using tethered space tug. Acta Astronaut 91:149-156

34. Benvenuto R, Salvi S, Lavagna M (2015) Dynamics analysis and GNC design of flexible systems for space debris active removal. Acta Astronaut 110:247-265
35. FET OPEN project, Electrodynamic tether technology for passive consumable-less deorbit kit (E.T.PACK), No. 828902, 1/3/201931/5/2022, https://etpack.eu/

36. Tarabini Castellani L, Urgoiti E, Sánchez-Arriaga G, Naghdi S, Lorenzini EC, Tajmar M et al (2019) Low work-function tether deorbit kit. LPICo 2109:6173

37. Sánchez-Arriaga G, Naghdi S, Wätzig K, Schilm J, Lorenzini EC, Tajmar M et al (2020) The E.T.PACK project: towards a fully passive and consumable-less deorbit kit based on low-work-function tether technology. Acta Astronaut. Printed on line

38. Sarego G, Olivieri L, Valmorbida A, Brunello A, Lorenzini EC et al. Deployment requirements for deorbiting electrodynamic tether technology. In: Proceedings of the aerospace Europe conference 2020, Bordeaux, France, 25-28 Feb 2020

39. Mantellato R, Pertile M, Colombatti G, Lorenzini E (2013) Analysis of passive system to damp the libration of electrodynamic tethers for deorbiting. In: AIAA Space2013 conference and exposition, 2013, p 5390

40. Mantellato R, Valmorbida A, Lorenzini EC (2015) Thrustaided librating deployment of tape tethers. J Spacecr Rockets 52(5):1395-1406

41. Lorenzini E, Mantellato R (2016) Electrodynamic tethers in space: dynamical issues, solutions and performance. In: Proceedings of the 14th spacecraft charging technology conference, ESA/ESTEC, Netherlands, 4-8 April 2016

42. Dobrowolny M, Stone NH (1994) A technical overview of TSS1: the first tethered-satellite system mission. Il Nuovo Cimento C 17(1):1-12

43. Bettanini C, Lorenzini EC, Colombatti G, Aboudan A, Massironi M (2018) CUTIE: a cubesats tether-inserted mission for moon exploration. Acta Astronaut 152:580-587

44. Petrillo D, Buonuomo M, Cavinato A, Chiarotti F, Gaino M, Branz F, Olivieri L, Sansone F, Borst G, Francesconi A (2015) Flexible electromagnetic leash docking system (FELDs) experiment from design to microgravity testing. In: 66th IAC, Jerusalem, 12-16 October 2015

45. Grassi G, Gloder A, Pellegrina L, Pezzato M, Rossi A, Branz F, Duzzi M, Mantellato R, Olivieri L, Sansone F, Lorenzini EC, Francesconi A (2017) An innovative space tether deployer with retrieval capability: design and test of STAR experiment. In: Proceedings of the 68th IAC, Adelaide, Australia

46. Coffey S, Carroll JA, Oppenheimer P et al (2019) Separation system and burn wire release mechanism for tethered spacecraft. US Patent 10,266,284

47. Mantellato R (2015) Investigation of deorbiting systems using passive electrodynamic propulsion. $\mathrm{PhD}$ thesis

48. Marshall LS, Geiger RV (1995) Deployer performance results for the TSS-1 mission. https://ntrs.nasa.gov/search.jsp?R=19970 001737

49. Vaughn J, Curtis L, Gilchrist B, Bilen S, Lorenzini E (2004) Review of the proSEDS electrodynamic tether mission development. In: 40th AIAA/ASME/SAE/ASEE joint propulsion conference and exhibit, Fort Lauderdale, Florida, USA, p 3501

50. Kruijff M, van der Heide EJ (2008) Qualification and in-flight demonstration of a European tether deployment system on YES2. Acta Astronaut 64(9-10):882-905

51. Krujiff M, van der Heide EJ, Ockels WJ, Gill E (2008) First mission results of the YES2 tethered SpaceMail experiment. In: AIAA-2008-7385, proceedings of the AIAA/AAS astrodynamics specialist conference, Honolulu, Hawaii, USA

52. Carroll AJ (1993) SEDS deployer design and flight performance. In: Proceedings of AIAA space programs and technologies conference and exhibit, Huntsville, AL, USA. https://doi. org/10.2514/6.1993-4764 
53. Lorenzini EC, Bortolami SB, Rupp CC, Angrilli F (1996) Control and flight performance of tethered satellite small expendable deployment system-II. J Guid Control Dyn 19(5):1148-1156. https://doi.org/10.2514/3.21757

54. Yu B, Geng L, Wen H, Chen T, Jin D (2018) Ground-based experiments of tether deployment subject to analytical control law. Acta Astronaut 151:253-259

55. Carroll JA (2019) In: TEPCE presentation at the first orbital debris conference (IOC-2019) Houston, Texas, USA, 9-12 Dec 2019

56. Francesconi A, Giacomuzzo C, Branz F, Lorenzini EC (2013) Survivability to hypervelocity impacts of electrodynamic tape tethers for deorbiting spacecraft in LEO. In: 6th European conference on space debris, April 22-25, Darmstadt, Germany

57. Valmorbida A, Mazzucato M, Tronco S, Pertile M, Lorenzini EC (2017) Design of a ground-based facility to reproduce satellite relative motions. In: 2017 IEEE MetroAeroSpace, Padua, Italy, 2017, pp 468-473

58. Valmorbida A, Mazzucato M, Aboudan A, Tronco S (2014) Test of attitude control maneuvers with a satellite formation flying testbed. In: 2014 IEEE MetroAeroSpace, Benevento, 29-30 May 2014, pp 439-444

59. Valmorbida A, Mazzucato M, Tronco S, Debei S, Lorenzini E (2015) SPARTANS - a cooperating spacecraft testbed for autonomous proximity operations experiments. In: IEEE instrumentation and measurement technology conference, vol 2015-July 2015

60. Valmorbida A, Mazzucato M, Pertile M (2020) Calibration procedures of a vision-based system for relative motion estimation between satellites flying in proximity. Measurement 151:107161. ISSN:0263-2241 